\title{
Higher-Order Minimizers and Generalized $(F, \rho)$-Convexity in Nonsmooth Vector Optimization over Cones
}

\author{
S. K. Suneja ${ }^{1}$, Sunila Sharma ${ }^{1}$, Malti Kapoor ${ }^{2 *}$ \\ ${ }^{1}$ Department of Mathematics, Miranda House, University of Delhi, Delhi, India \\ ${ }^{2}$ Department of Mathematics, Motilal Nehru College, University of Delhi, Delhi, India \\ Email: ${ }^{*}$ maltikapoor1@gmail.com
}

Received 1 November 2014; revised 29 November 2014; accepted 16 December 2014

Copyright (C) 2015 by authors and Scientific Research Publishing Inc.

This work is licensed under the Creative Commons Attribution International License (CC BY).

http://creativecommons.org/licenses/by/4.0/

(c) () Open Access

\begin{abstract}
In this paper, we introduce the concept of a (weak) minimizer of order $\boldsymbol{k}$ for a nonsmooth vector optimization problem over cones. Generalized classes of higher-order cone-nonsmooth $(F, \rho)$ convex functions are introduced and sufficient optimality results are proved involving these classes. Also, a unified dual is associated with the considered primal problem, and weak and strong duality results are established.
\end{abstract}

\section{Keywords}

Nonsmooth Vector Optimization over Cones, (Weak) Minimizers of Order $k$, Nonsmooth $(F, \rho)$-Convex Function of Order $k$

\section{Introduction}

It is well known that the notion of convexity plays a key role in optimization theory [1] [2]. In the literature, various generalizations of convexity have been considered. One such generalization is that of a $\rho$-convex function introduced by Vial [3]. Hanson and Mond [4] defined the notion of an F-convex function. As an extended unification of the two concepts, Preda [5] introduced the concept of a $(F, \rho)$-convex function. Antczak gave the notion of a locally Lipschitz $(F, \rho)$-convex scalar function of order $k[6]$ and a differentiable $(F, \rho)$ convex vector function of order 2 [7].

L. Cromme [8] defined the concept of a strict local minimizer of order $k$ for a scalar optimization problem. This concept plays a fundamental role in convergence analysis of iterative numerical methods [8] and in stability

"Corresponding author.

How to cite this paper: Suneja, S.K., Sharma, S. and Kapoor, M. (2015) Higher-Order Minimizers and Generalized (F, $\rho$ )Convexity in Nonsmooth Vector Optimization over Cones. Applied Mathematics, 6, 7-19.

http://dx.doi.org/10.4236/am.2015.61002 
results [9]. The definition of a strict local minimizer of order 2 is generalized to the vectorial case by Antczak [7].

Recently, Bhatia and Sahay [10] introduced the concept of a higher-order strict minimizer with respect to a nonlinear function for a differentiable multiobjective optimization problem. They proved various sufficient optimality and mixed duality results involving generalized higher-order strongly invex functions.

The main purpose of this paper is to extend the concept of a higher-order minimizer to a nonsmooth vector optimization problem over cones. The paper is organized as follows. We begin in Section 2 by recalling some known concepts in the literature. We then define the notion of a (weak) minimizer of order $k$ for a nonsmooth vector optimization problem over cones. Thereafter, we introduce various new generalized classes of conenonsmooth $(F, \rho)$-convex functions of higher-order. In Section 3, we study several optimality conditions for higher-order minimizers via the introduced classes of functions. In Section 4, we associate a unified dual to the considered problem and establish weak and strong duality results.

\section{Preliminaries and Definitions}

Let $S \subseteq \mathbf{R}^{n}$ be a nonempty open subset of $\mathbf{R}^{n}$. Let $K \subseteq \mathbf{R}^{m}$ be a closed convex cone with nonempty interior and let int $K$ denote the interior of $K$. The dual cone $K^{*}$ of $K$ is defined as

$$
K^{*}=\left\{y^{*} \in \mathbf{R}^{m}:\left\langle y, y^{*}\right\rangle \geq 0 \text { for all } y \in K\right\} .
$$

The strict positive dual cone $K^{s^{*}}$ of $K$ is given by

$$
K^{s^{*}}=\left\{y^{*} \in \mathbf{R}^{m}:\left\langle y, y^{*}\right\rangle>0 \text { for all } y \in K \backslash\{0\}\right\} .
$$

A function $\psi: S \rightarrow \mathbf{R}$ is said to be locally Lipschitz at a point $u \in S$ if for some $l>0$, $\|\psi(x)-\psi(\bar{x})\| \leq l\|x-\bar{x}\| \quad \forall x, \bar{x}$ within a neighbourhood of $u$.

A function $\psi$ is said to be locally Lipschitz on $S$ if it is locally Lipschitz at each point of $S$.

Definition 2.1. [11] Let $\psi: S \rightarrow \mathbf{R}$ be a locally Lipschitz function, then $\psi^{0}(u ; v)$ denotes the Clarke's generalized directional derivative of $\psi$ at $u \in S$ in the direction $v$ and is defined as

$$
\psi^{0}(u ; v)=\limsup _{\substack{y \rightarrow u \\ t \rightarrow 0^{+}}} \frac{\psi(y+t v)-\psi(y)}{t} .
$$

The Clarke's generalized gradient of $\psi$ at $u$ is denoted by $\partial \psi(u)$ and is defined as

$$
\partial \psi(u)=\left\{\xi \in \mathbf{R}^{n}: \psi^{0}(u ; v) \geq\langle\xi, v\rangle \text { for all } v \in \mathbf{R}^{n}\right\} .
$$

Let $f: S \rightarrow \mathbf{R}^{m}$ be a vector valued function given by $f=\left(f_{1}, f_{2}, \cdots, f_{m}\right)^{t}, f_{i}: S \rightarrow \mathbf{R}$. Then $f$ is said to be locally Lipschitz on $S$ if each $f_{i}$ is locally Lipschitz on $S$. The generalized directional derivative of a locally Lipschitz function $f: S \rightarrow \mathbf{R}^{m}$ at $u \in S$ in the direction $v$ is given by

$$
f^{0}(u ; v)=\left(f_{1}^{0}(u ; v), f_{2}^{0}(u ; v), \cdots, f_{m}^{0}(u ; v)\right)^{t} .
$$

The generalized gradient of $f$ at $u$ is the set

$$
\partial f(u)=\partial f_{1}(u) \times \cdots \times \partial f_{m}(u),
$$

where $\partial f_{i}(u)$ is the generalized gradient of $f_{i}$ at $u$ for $i=1,2, \cdots, m$.

Every element $A=\left(A_{1}, \cdots, A_{m}\right)^{l} \in \partial f(u)$ is a continuous linear operator from $\mathbf{R}^{n}$ to $\mathbf{R}^{m}$ and $A u=\left(A_{1}^{t} u, \cdots, A_{m}^{t} u\right)^{t} \in \mathbf{R}^{m}$ for all $u \in S$.

A functional $F: S \times S \times \mathbf{R}^{n} \rightarrow \mathbf{R}$ is sublinear with respect to the third variable if, for all $(x, u) \in S \times S$,

(i) $F\left(x, u ; A_{1}+A_{2}\right) \leq F\left(x, u ; A_{1}\right)+F\left(x, u ; A_{2}\right)$ for all $A_{1}, A_{2} \in \mathbf{R}^{n}$, and

(ii) $F(x, u ; \alpha A)=\alpha F(x, u ; A)$ for all $\alpha \in \mathbf{R}_{+}$. 
(i) and (ii) together imply $F(x, u ; 0)=0$.

We consider the following nonsmooth vector optimization problem

$$
\begin{array}{ll}
\text { (NVOP) } & \text { K-minimize } f(x) \\
& \text { subject to }-g(x) \in Q,
\end{array}
$$

where $f=\left(f_{1}, \cdots, f_{m}\right)^{t}: S \rightarrow \mathbf{R}^{m}, g=\left(g_{1}, \cdots, g_{p}\right)^{t}: S \rightarrow \mathbf{R}^{p}, K$ and $Q$ are closed convex cones with nonempty interiors in $\mathbf{R}^{m}$ and $\mathbf{R}^{p}$ respectively. We assume that $f_{i}$ for each $i \in\{1, \cdots, m\}$ and $g_{j}$ for each $j \in\{1, \cdots, p\}$ are locally Lipschitz on $S$.

Let $S_{0}=\{x \in S:-g(x) \in Q\}$ denote the set of all feasible solutions of (NVOP).

The following solution concepts are well known in the literature of vector optimization theory.

Definition 2.2. A point $\bar{x} \in S_{0}$, is said to be

(i) a weak minimizer (weakly efficient solution) of (NVOP) if for every $x \in S_{0}$,

$$
f(x)-f(\bar{x}) \notin-\mathrm{int} K \text {; }
$$

(ii) a minimizer (efficient solution) of (NVOP) if for every $x \in S_{0}$,

$$
f(x)-f(\bar{x}) \notin-K \backslash\{0\} .
$$

With the idea of analyzing the convergence and stability of iterative numerical methods, L. Cromme [8] introduced the notion of a "strict local minimizer of order $k$ ". As a recent advancement on this platform, Bhatia and Sahay [10] defined the concept of a higher-order strict minimizer with respect to a nonlinear function for a differentiable multiobjective optimization problem. We now generalize this concept and give the definition of a higher-order (weak) minimizer with respect to a function $\omega$ for a nonsmooth vector optimization problem over cones.

Definition 2.3. A point $\bar{x} \in S_{0}$ is said to be

(i) a weak minimizer of order $k \geq 1$ for (NVOP) with respect to $\omega$, if there exists a vector $\beta \in$ int $K$ such that, for every $x \in S_{0}$

$$
f(x)-f(\bar{x})-\beta\|\omega(x, \bar{x})\|^{k} \notin-\operatorname{int} K ;
$$

(ii) a minimizer of order $k \geq 1$ for (NVOP) with respect to $\omega$, if there exists a vector $\beta \in$ int $K$ such that, for every $x \in S_{0}$

$$
f(x)-f(\bar{x})-\beta\|\omega(x, \bar{x})\|^{k} \notin-K \backslash\{0\} .
$$

Remark 2.1. (1) If $f$ is a scalar valued function, $K=\mathbf{R}_{+}$and $\omega(x, \bar{x})=x-\bar{x}$, the definition of a weak minimizer of order $k$ reduces to the definition of a strict minimizer of order $k$ (see [8] [9] [12] [13]).

(2) If $K=\mathbf{R}_{+}^{m}, \quad k=2$ and $\omega(x, \bar{x})=x-\bar{x}$, the definition of a (weak) minimizer of order $k$ becomes the definition of a vector strict global (weak) minimizer of order 2 given by Antczak [7].

(3) If $K=\mathbf{R}_{+}^{m}$ the definition of a weak minimizer of order $k$ reduces to the definition of a strict minimizer of order $k$ given by Bhatia and Sahay [10].

Remark 2.2. (1) Clearly a minimizer of order $k$ for (NVOP) with respect to $\omega$ is also a weak minimizer of order $k$ for (NVOP) with respect to the same $\omega$.

(2) A direct implication of the fact that $\beta \in \operatorname{int} K$ is that, a (weak) minimizer of order $k$ for (NVOP) with respect to $\omega$ is a (weak) minimizer for (NVOP).

(3) Note that if $\bar{x}$ is a (weak) minimizer of order $k$ for (NVOP) with respect to $\omega$, then for all $\ell>k$, it is also a (weak) minimizer of order $\ell$ for (NVOP) with respect to the same $\omega$.

In the sequel, for a vector function $f: S \rightarrow \mathbf{R}^{m}$ and $A=\left(A_{1}, \cdots, A_{m}\right)^{t} \in \partial f(u), F(x, u ; A)$ denotes the vector $\left(F\left(x, u ; A_{1}\right), \cdots, F\left(x, u ; A_{m}\right)\right)^{t}$.

We now define various classes of nonsmooth $(F, \rho)$-convex functions of higher-order over cones.

Definition 2.4. A locally Lipschitz function $f: S \rightarrow \mathbf{R}^{m}$ is said to be K-nonsmooth $(F, \rho)$-convex of order $k$ with respect to $\omega$ at $u \in S$ on $S$ if there exist a sublinear (with respect to the third variable) functional 
$F: S \times S \times \mathbf{R}^{n} \rightarrow \mathbf{R}$ and a vector $\rho=\left(\rho_{1}, \rho_{2}, \cdots, \rho_{m}\right) \in \mathbf{R}^{m}$ such that, for each $A \in \partial f(u)$ and all $x \in S$

$$
f(x)-f(u)-F(x, u ; A)-\rho\|\omega(x, u)\|^{k} \in K .
$$

If the above relation holds for every $u \in S$ then $f$ is said to be $K$-nonsmooth $(F, \rho)$-convex of order $k$ with respect to $\omega$ on $S$.

Remark 2.3. (1) If $f$ is a scalar valued function and $K=\mathbf{R}_{+}$, the above definition reduces to the definition of a (locally Lipschitz) $(F, \rho)$-convex function of order $k$ with respect to $\omega$ given by Antczak [6].

(2) If $f$ is a differentiable function, $K=\mathbf{R}_{+}^{m}, k=2$ and $\omega(x, \bar{x})=x-\bar{x}$ the definition of a $K$-nonsmooth $(F, \rho)$-convex function of order $k$ with respect to $\omega$ becomes the definition of a vector $(F, \rho)$-convex function of order 2 given in [7].

(3) If $K=\mathbf{R}_{+}^{m}, F(x, u ; A)=A \eta(x, u)$ for some function $\eta: S \times S \rightarrow \mathbf{R}^{n}$ and $k=2, K$-nonsmooth $(F, \rho)$ convexity of order $k$ with respect to $\omega$ reduces to $\rho-(\eta, \theta)$-invexity, where $\omega(x, \bar{x})=\theta(x, \bar{x})$, introduced by Nahak and Mohapatra [14].

(4) If $f$ is a differentiable function, $K=\mathbf{R}_{+}^{m}$ and $F(x, u ; a)=a^{t} \eta(x, u), a \in \mathbf{R}^{n}$, for some function $\eta: S \times S \rightarrow \mathbf{R}^{n}$, the above definition becomes the definition of a higher-order strongly invex function given by Bhatia and Sahay [10].

Definition 2.5. A locally Lipschitz function $f: S \rightarrow \mathbf{R}^{m}$ is said to be $K$-nonsmooth $(F, \rho)$-pseudoconvex type I of order $k$ with respect to $\omega$ at $u \in S$ on $S$ if there exist a sublinear (with respect to the third variable) functional $F: S \times S \times \mathbf{R}^{n} \rightarrow \mathbf{R}$ and a vector $\rho \in \mathbf{R}^{m}$ such that, for each $A \in \partial f(u)$ and all $x \in S$,

$$
-F(x, u ; A) \notin \operatorname{int} K \Rightarrow-\left[f(x)-f(u)-\rho\|\omega(x, u)\|^{k}\right] \notin \operatorname{int} K .
$$

Equivalently,

$$
f(x)-f(u)-\rho\|\omega(x, u)\|^{k} \in-\operatorname{int} K \Rightarrow F(x, u ; A) \in-\operatorname{int} K .
$$

If $f$ is $K$-nonsmooth $(F, \rho)$-pseudoconvex type I of order $k$ with respect to $\omega$ at every $u \in S$ then $f$ is said to be $K$-nonsmooth $(F, \rho)$-pseudoconvex type I of order $k$ with respect to $\omega$ on $S$.

Clearly, if $f$ is $K$-nonsmooth $(F, \rho)$-convex of order $k$ with respect to $\omega$, then $f$ is $K$-nonsmooth $(F, \rho)$ pseudoconvex type I of order $k$ with respect to the same $\omega$, however the converse may not be true as shown by the following example.

Example 2.1. Consider the following nonsmooth function $f: S \rightarrow \mathbf{R}^{2}, S=(-2,2) \subseteq \mathbf{R}, f(x)=\left(f_{1}(x), f_{2}(x)\right)$ and $K=\{(x, y): x \geq 0, y \leq x\}$

$$
f_{1}(x)=\left\{\begin{array}{ll}
-2 x, & x<0 \\
-x^{6}-x, & x \geq 0
\end{array} \quad f_{2}(x)= \begin{cases}x^{3}-x / 2, & x<0 \\
-x / 3, & x \geq 0\end{cases}\right.
$$

Here $\partial f_{1}(0)=[-2,-1]$ and $\partial f_{2}(0)=\left[-\frac{1}{2},-\frac{1}{3}\right]$.

Define $F: S \times S \times \mathbf{R} \rightarrow \mathbf{R}$ as

$$
F(x, u ; a)=a(\sqrt{x}-\sqrt{u}) .
$$

Let $\omega: S \times S \rightarrow R$ be given by $\omega(x, u)=x^{2}-u^{2}, k=3$ and $\rho=(-1,0)$.

Then, at $u=0$.

$$
f(x)-f(u)-\rho\|\omega(x, u)\|^{k} \in-\operatorname{int} K \Rightarrow x>0 \Rightarrow F(x, u ; A) \in-\operatorname{int} K,
$$

for every $x \in S$ and $A \in \partial f(0)$.

Hence, $f$ is $K$-nonsmooth $(F, \rho)$-pseudoconvex type I of order 3 with respect to $\omega$ at $u$ on $S$.

However, for $x=1$ and $A=(-1,-1 / 2)$.

$$
f(x)-f(u)-F(x, u ; A)-\rho\|\omega(x, u)\|^{k} \notin K,
$$


so that $f$ is not $K$-nonsmooth $(F, \rho)$-convex of order 3 at $u$ on $S$.

Definition 2.6. A locally Lipschitz function $f: S \rightarrow \mathbf{R}^{m}$ is said to be $K$-nonsmooth $(F, \rho)$-pseudoconvex type II of order $k$ with respect to $\omega$ at $u \in S$ on $S$ if there exist a sublinear (with respect to the third variable) functional $F: S \times S \times \mathbf{R}^{n} \rightarrow \mathbf{R}$ and a vector $\rho \in \mathbf{R}^{m}$ such that, for each $A \in \partial f(u)$ and all $x \in S$,

$$
-\left[F(x, u ; A)+\rho\|\omega(x, u)\|^{k}\right] \notin \operatorname{int} K \Rightarrow-[f(x)-f(u)] \notin \operatorname{int} K
$$

Equivalently,

$$
f(x)-f(u) \in-\operatorname{int} K \Rightarrow\left[F(x, u ; A)+\rho\|\omega(x, u)\|^{k}\right] \in-\operatorname{int} K .
$$

If the above relation holds for every $u \in S$, then $f$ is said to be $K$-nonsmooth $(F, \rho)$-pseudoconvex type II of order $k$ with respect to $\omega$ on $S$.

We now give an example to show that a $K$-nonsmooth $(F, \rho)$-pseudoconvex type II function of order $k$ with respect to $\omega$ may fail to be a $K$-nonsmooth $(F, \rho)$-convex function of order $k$ with respect to $\omega$.

Example 2.2. Consider the following nonsmooth function $f: S \rightarrow \mathbf{R}^{2}, S=(0,2) \subseteq \mathbf{R}, f(x)=\left(f_{1}(x), f_{2}(x)\right)$ and $K=\{(x, y): x \leq 0, y \geq x\}$

$$
f_{1}(x)=\left\{\begin{array}{ll}
-x, & x \leq 1 \\
-x^{2}, & x>1
\end{array}, \quad f_{2}(x)= \begin{cases}x / 4, & x \leq 1 \\
(x-1 / 2)^{2}, & x>1\end{cases}\right.
$$

Here $\partial f_{1}(1)=[-2,-1]$ and $\partial f_{2}(1)=\left[\frac{1}{4}, 1\right]$.

Let $F: S \times S \times \mathbf{R} \rightarrow \mathbf{R}$ be given by $F(x, u ; a)=a\left(\mathrm{e}^{x}-\mathrm{e}^{u}\right)$.

$$
\omega(x, u)=x^{2}-u^{2}-\frac{9}{16} \text { and } \rho=(1,-1) \text {. }
$$

Then, at $u=1$,

$$
f(x)-f(u) \in-\operatorname{int} K \Rightarrow x \leq 1 \Rightarrow F(x, u ; A)+\rho\|\omega(x, u)\|^{k} \in-\operatorname{int} K,
$$

for every $k \geq 1, \quad x \in S$ and $A \in \partial f(1)$.

Therefore, $f$ is $K$-nonsmooth $(F, \rho)$-pseudoconvex type II of order $k \geq 1$ with respect to $\omega$ at $u$ on $S$.

However, for $x=5 / 4$ and $A=\left(-2, \alpha_{2}\right), \alpha_{2} \in[1 / 4,1]$,

$$
f(x)-f(u)-F(x, u ; A)-\rho\|\omega(x, u)\|^{k} \notin K .
$$

Thus, $f$ is not $K$-nonsmooth $(F, \rho)$-convex of any order $k$ with respect to $\omega$ at $u$ on $S$.

Definition 2.7. A locally Lipschitz function $f: S \rightarrow \mathbf{R}^{m}$ is said to be $K$-nonsmooth $(F, \rho)$-quasiconvex type I of order $k$ with respect to $\omega$ at $u \in S$ on $S$ if there exist a sublinear (with respect to the third variable) functional $F: S \times S \times R^{n} \rightarrow R$ and a vector $\rho \in R^{m}$ such that, for each $A \in \partial f(u)$ and all $x \in S$,

$$
[f(x)-f(u)] \notin \operatorname{int} K \Rightarrow-\left[F(x, u ; A)+\rho\|\omega(x, u)\|^{k}\right] \in K .
$$

If the above relation holds at every $u \in S$, then $f$ is said to be $K$-nonsmooth $(F, \rho)$-quasiconvex type I of order $k$ with respect to $\omega$ on $S$.

Definition 2.8. A locally Lipschitz function $f: S \rightarrow \mathbf{R}^{m}$ is said to be $K$-nonsmooth $(F, \rho)$-quasiconvex type II of order $k$ with respect to $\omega$ at $u \in S$ on $S$ if there exist a sublinear (with respect to the third variable) functional $F: S \times S \times \mathbf{R}^{n} \rightarrow \mathbf{R}$ and a vector $\rho \in \mathbf{R}^{m}$ such that, for each $A \in \partial f(u)$ and all $x \in S$,

$$
\left[f(x)-f(u)-\rho\|\omega(x, u)\|^{k}\right] \notin \operatorname{int} K \Rightarrow-F(x, u ; A) \in K .
$$

If $f$ is $K$-nonsmooth $(F, \rho)$-quasiconvex type II of order $k$ with respect to $\omega$ at every $u \in S$, then $f$ is said 
to be $K$-nonsmooth $(F, \rho)$-quasiconvex type II of order $k$ with respect to $\omega$ on $S$.

Remark 2.4. When $f$ is a differentiable function, $K=\mathbf{R}_{+}^{m}$ and $F(x, u ; a)=a^{t} \eta(x, u), a \in \mathbf{R}^{n}$ for some function $\eta: S \times S \rightarrow \mathbf{R}^{n}$, Definition $2.4-2.7$ take the form of the corresponding definitions given by Bhatia and Sahay [10].

\section{Optimality}

In this section, we obtain various nonsmooth Fritz John type and Karush-Kuhn-Tucker (KKT) type necessary and sufficient optimality conditions for a feasible solution to be a (weak) minimizer of order $k$ for (NVOP).

On the lines of Craven [15] we define Slater-type cone constraint qualification as follows:

Definition 3.1. The problem (NVOP) is said to satisfy Slater-type cone constraint qualification at $\bar{x}$ if, for all $B \in \partial g(\bar{x})$, there exists a vector $\xi \in R^{n}$ such that $B \xi \in-$ int $Q$.

Remark 3.1. The following inclusion relation is worth noticing.

$$
\begin{aligned}
& \text { For } \lambda=\left(\lambda_{1}, \cdots, \lambda_{m}\right)^{t} \in \mathbf{R}^{m} \text { and } \mu=\left(\mu_{1}, \cdots, \mu_{p}\right)^{t} \in \mathbf{R}^{p} \text {, } \\
& \partial\left(\lambda^{t} f+\mu^{t} g\right)(\bar{x})=\partial\left(\sum_{i=1}^{m} \lambda_{i} f_{i}+\sum_{j=1}^{p} \mu_{j} g_{j}\right)(\bar{x}) \\
& \subseteq \partial\left(\sum_{i=1}^{m} \lambda_{i} f_{i}\right)(\bar{x})+\partial\left(\sum_{j=1}^{p} \mu_{j} g_{j}\right)(\bar{x}) \\
& \subseteq \sum_{i=1}^{m} \lambda_{i} \partial f_{i}(\bar{x})+\sum_{j=1}^{p} \mu_{j} \partial g_{j}(\bar{x}) \\
& =\left(\partial f(\bar{x})^{t} \lambda+\partial g(\bar{x})^{t} \mu\right) \text {. }
\end{aligned}
$$

Thus,

$$
\partial\left(\lambda^{t} f+\mu^{t} g\right)(\bar{x}) \subseteq\left(\partial f(\bar{x})^{t} \lambda+\partial g(\bar{x})^{t} \mu\right) .
$$

Since a weak minimizer of order $k \geq 1$ for (NVOP) is a weak minimizer for (NVOP), the following nonsmooth Fritz John type necessary optimality conditions can be easily obtained from Craven [15].

Theorem 3.1. If a vector $\bar{x} \in S_{0}$ is a weak minimizer of order $k$ with respect to $\omega$ for (NVOP) with $S=\mathbf{R}^{n}$, then there exist Lagrange multipliers $\bar{\lambda} \in K^{*}$ and $\bar{\mu} \in Q^{*}$ not both zero, such that

$$
\begin{gathered}
0 \in \partial\left(\bar{\lambda}^{t} f+\bar{\mu}^{t} g\right)(\bar{x}) \\
\bar{\mu}^{t} g(\bar{x})=0 .
\end{gathered}
$$

The necessary nonsmooth KKT type optimality conditions for (NVOP) can be given in the following form.

Theorem 3.2. If a vector $\bar{x} \in S_{0}$ is a weak minimizer of order $k$ with respect to $\omega$ for (NVOP) with $S=\mathbf{R}^{n}$ and if Slater-type cone constraint qualification holds at $\bar{x}$, then there exist Lagrange multipliers $\bar{\lambda} \in K^{*} \backslash\{0\}$ and $\bar{\mu} \in Q^{*}$, such that

$$
\begin{gathered}
0 \in \partial\left(\bar{\lambda}^{t} f+\bar{\mu}^{t} g\right)(\bar{x}) \\
\bar{\mu}^{t} g(\bar{x})=0 .
\end{gathered}
$$

Proof. Assume that $\bar{x} \in S_{0}$ is a weak minimizer of order $k$ with respect to $\omega$ for (NVOP), then by Theorem 3.1 there exist $\bar{\lambda} \in K^{*}$ and $\bar{\mu} \in Q^{*}$, not both zero, such that (3) and (4) hold.

If possible, suppose $\bar{\lambda}=0$. Then, $\bar{\mu} \neq 0$ and (3) reduces to

$$
0 \in \partial\left(\bar{\mu}^{t} g\right)(\bar{x}) \subseteq \partial g(\bar{x})^{t} \bar{\mu} .
$$

So there exists $\bar{B} \in \partial g(\bar{x})$ such that

$$
\bar{B}^{t} \bar{\mu}=0 \text {. }
$$


Now, since Slater-type cone constraint qualification holds at $\bar{x}$, we have for all $B \in \partial g(\bar{x})$, there exists a vector $\xi \in R^{n}$ such that $B \xi \in-$ int $Q$. Since $\bar{\mu} \in Q^{*} \backslash\{0\}$, we get $\bar{\mu}^{t} B \xi<0$. In particular, $\bar{\mu}^{t} \bar{B} \xi<0$. On the contrary (5) implies $\xi^{t} \bar{B}^{t} \bar{\mu}=0$. This contradiction justifies $\bar{\lambda} \neq 0$.

Now, we give sufficient optimality conditions for a feasible solution to be a higher-order (weak) minimizer for (NVOP).

Theorem 3.3. Let $\bar{x}$ be a feasible solution for (NVOP) and suppose there exist vectors $\bar{\lambda} \in K^{*}, \bar{\lambda}>0$ and $\bar{\mu} \in Q^{*}, \bar{\mu} \geq 0$ such that

$$
\begin{gathered}
0 \in\left(\partial f(\bar{x})^{t} \bar{\lambda}+\partial g(\bar{x})^{t} \bar{\mu}\right) \\
\bar{\mu}^{t} g(\bar{x})=0 .
\end{gathered}
$$

Further, assume that $f$ is $K$-nonsmooth $(F, \rho)$-convex of order $k$ with respect to $\omega$ at $\bar{X}$ on $S_{0}$ and $g$ is $Q$-nonsmooth $(F, \sigma)$-convex of order $k$ with respect to the same $\omega$ at $\bar{x}$ on $S_{0}$. If $\rho \in \operatorname{int} K$ and $\sigma \in Q$, then $\bar{x}$ is a weak minimizer of order $k$ with respect to $\omega$ for (NVOP).

Proof. Assume on the contrary that $\bar{x}$ is not a weak minimizer of order $k$ with respect to $\omega$ for (NVOP). Then, for any $\beta \in \operatorname{int} K$, there exists a vector $\hat{x} \in S_{0}$ such that,

$$
f(\hat{x})-f(\bar{x})-\beta\|\omega(\hat{x}, \bar{x})\|^{k} \in-\operatorname{int} K .
$$

As $\rho \in \operatorname{int} K$, the above relation holds in particular for $\beta=\rho$, so that we have

$$
f(\hat{x})-f(\bar{x})-\rho\|\omega(\hat{x}, \bar{x})\|^{k} \in-\operatorname{int} K .
$$

As (6) holds, there exist $\bar{A} \in \partial f(\bar{x})$ and $\bar{B} \in \partial g(\bar{x})$ such that

$$
\bar{A}^{t} \bar{\lambda}+\bar{B}^{t} \bar{\mu}=0 \text {. }
$$

Since $f$ is $K$-nonsmooth $(F, \rho)$-convex of order $k$ with respect to $\omega$ at $\bar{x}$ on $S_{0}$, we have

$$
f(\hat{x})-f(\bar{x})-F(\hat{x}, \bar{x} ; \bar{A})-\rho\|\omega(\hat{x}, \bar{x})\|^{k} \in K .
$$

Adding (8) and (10), we get

$$
-F(\hat{x}, \bar{x} ; \bar{A}) \in \operatorname{int} K
$$

As $\lambda \in K^{*} \backslash\{0\}$, we obtain

$$
\bar{\lambda}^{t} F(\hat{x}, \bar{x} ; \bar{A})<0 .
$$

Also, since $g$ is $Q$-nonsmooth $(F, \sigma)$ convex of order $k$ with respect to $\omega$ at $\bar{x}$ on $S_{0}$ and $\bar{\mu} \in Q^{*}$, we have

$$
\bar{\mu}^{t}\left[g(\hat{x})-g(\bar{x})-F(\hat{x}, \bar{x} ; \bar{B})-\sigma\|\omega(\hat{x}, \bar{x})\|^{k}\right] \geq 0 .
$$

However, $\hat{x} \in S_{0}, \quad \bar{\mu} \in Q^{*}$ and (7) together give

$$
\bar{\mu}^{t}\left[F(\hat{x}, \bar{x} ; \bar{B})+\sigma\|\omega(\hat{x}, \bar{x})\|^{k}\right] \leq 0 .
$$

Adding (11) and (12), we get

$$
\bar{\lambda}^{t} F(\hat{x}, \bar{x} ; \bar{A})+\bar{\mu}^{t} F(\hat{x}, \bar{x} ; \bar{B})+\bar{\mu}^{t} \sigma\|\omega(\hat{x}, \bar{x})\|^{k}<0,
$$

which implies that

$$
\sum_{i} \bar{\lambda}_{i} F\left(\hat{x}, \bar{x} ; \bar{A}_{i}\right)+\sum_{j} \bar{\mu}_{j} F\left(\hat{x}, \bar{x} ; \bar{B}_{j}\right)+\bar{\mu}^{t} \sigma\|\omega(\hat{x}, \bar{x})\| \|^{k}<0 .
$$


Using sublinearity of $F$ under the assumption $\bar{\lambda}>0$ and $\bar{\mu} \geq 0$, we obtain

$$
F\left(\hat{x}, \bar{x} ; \bar{\lambda}^{t} \bar{A}+\bar{\mu}^{t} \bar{B}\right)+\bar{\mu}^{t} \sigma\|\omega(\hat{x}, \bar{x})\|^{k}<0,
$$

which on using (9) and (1), gives

$$
\bar{\mu}^{t} \sigma\|\omega(\hat{x}, \bar{x})\|^{k}<0 .
$$

This is impossible as $\bar{\mu} \in Q^{*}$ and $\sigma \in Q$, so that $\bar{\mu}^{t} \sigma \geq 0$, and norm is a non-negative function. Hence $\bar{x}$ is a weak minimizer of order $k$ with respect to $\omega$ for (NVOP).

Theorem 3.4. Suppose there exists a feasible solution $\bar{x}$ for (NVOP) and vectors $\bar{\lambda} \in K^{*}, \bar{\lambda}>0$ and $\bar{\mu} \in Q^{*}, \bar{\mu} \geq 0$ such that (6) and (7) hold. Moreover, assume that $f$ is $K$-nonsmooth $(F, \rho)$-pseudoconvex type I of order $k$ with respect to $\omega$ at $\bar{x}$ on $S_{0}$ and $g$ is $Q$-nonsmooth $(F, \sigma)$-quasiconvex type I of order $k$ with respect to the same $\omega$ at $\bar{x}$ on $S_{0}$. If $\rho \in \operatorname{int} K$ and $\sigma \in Q$, then $\bar{x}$ is a weak minimizer of order $k$ with respect to $\omega$ for (NVOP).

Proof: Let if possible, $\bar{x}$ be not a weak minimizer of order $k$ with respect to $\omega$ for (NVOP). Then, for any $\beta \in \operatorname{int} K$, there exists $\hat{x} \in S_{0}$ such that,

$$
f(\hat{x})-f(\bar{x})-\beta\|\omega(\hat{x}, \bar{x})\|^{k} \in-\operatorname{int} K .
$$

Since $\rho \in \operatorname{int} K$ taking, in particular, $\beta=\rho$ in the above relation, we obtain

$$
f(\hat{x})-f(\bar{x})-\rho\|\omega(\hat{x}, \bar{x})\|^{k} \in-\operatorname{int} K .
$$

As (6) holds, there exist $\bar{A} \in \partial f(\bar{x})$ and $\bar{B} \in \partial g(\bar{x})$ such that (9) holds.

Since $f$ is $K$-nonsmooth $(F, \rho)$-pseudoconvex type I of order $k$ with respect to $\omega$ at $\bar{x}$ on $S_{0}$, (13) implies

$$
F(\hat{x}, \bar{x} ; \bar{A}) \in-\text { int } K \text {. }
$$

As $\lambda \in K^{*} \backslash\{0\}$, we have

$$
\bar{\lambda}^{t} F(\hat{x}, \bar{x} ; \bar{A})<0 \text {. }
$$

Now, $\hat{x} \in S_{0}$ means $-g(\hat{x}) \in Q$, so that $\bar{\mu}^{t} g(\hat{x}) \leq 0$. This along with (7) gives

$$
\bar{\mu}^{t}\{g(\hat{x})-g(\bar{x})\} \leq 0 .
$$

If $\bar{\mu} \neq 0$, then (15) implies $g(\hat{x})-g(\bar{x}) \notin$ int $Q$.

Since $g$ is $Q$-nonsmooth $(F, \sigma)$-quasiconvex type I of order $k$ with respect to $\omega$ at $\bar{x}$ on $S_{0}$, therefore

$$
-\left[F(\hat{x}, \bar{x} ; \bar{B})+\sigma\|\omega(\hat{x}, \bar{x})\|^{k}\right] \in Q,
$$

so that

$$
\bar{\mu}^{t}\left[F(\hat{x}, \bar{x} ; \bar{B})+\sigma\|\omega(\hat{x}, \bar{x})\|^{k}\right] \leq 0
$$

If $\bar{\mu}=0$, then also (16) holds.

Now, proceeding as in Theorem 3.3, we get a contradiction. Hence, $\bar{x}$ is a weak minimizer of order $k$ with respect to $\omega$ for (NVOP).

Theorem 3.5. Assume that all the conditions of Theorem 3.3 (Theorem 3.4) hold with $\bar{\lambda} \in K^{s^{*}}, \bar{\lambda}>0$. Then $\bar{x}$ is a minimizer of order $k$ with respect to $\omega$ for (NVOP).

Proof: Let if possible, $\bar{x}$ be not a minimizer of order $k$ with respect to $\omega$ for (NVOP), then for any $\beta \in \operatorname{int} K$ there exists $\hat{x} \in S_{0}$ such that

$$
-\left[f(\hat{x})-f(\bar{x})-\beta\|\omega(\hat{x}, \bar{x})\|^{k}\right] \in K \backslash\{0\} .
$$


Proceeding on similar lines as in proof of Theorem 3.3 (Theorem3.4) and using (17) we have

$$
-F(\hat{x}, \bar{x} ; \bar{A}) \in K \backslash\{0\} .
$$

As $\bar{\lambda} \in K^{s^{*}}$, we get

$$
\bar{\lambda}^{t} F(\hat{x}, \bar{x} ; \bar{A})<0
$$

This leads to a contradiction as in Theorem 3.3 (Theorem 3.4). Hence, $\bar{x}$ is a minimizer of order $k$ with respect to $\omega$ for (NVOP).

\section{Unified Duality}

On the lines of Cambini and Carosi [16], we associate with our primal problem (NVOP), the following unified dual problem (NVUD).

(NVUD) $\quad K$-maximize $f(y)+\frac{l}{\lambda^{t} l}(1-\delta) \mu^{t} g(y)$

$$
\begin{array}{ll}
\text { subject to } & 0 \in \partial\left(\lambda^{t} f+\mu^{t} g\right)(y), \\
& \delta \mu^{t} g(y) \geq 0
\end{array}
$$

where $y \in S, \quad l \in \operatorname{int} K, \lambda \in K^{*} \backslash\{0\}, \mu \in Q^{*}$ and $\delta \in\{0,1\}$ is a 0 - 1 parameter.

Note that Wolfe dual and Mond-Weir dual can be obtained from (NVUD) on taking $\delta=0$ and $\delta=1$ respectively.

Definition 4.1. Given the problem (NVOP) and given a vector $l \in$ int $K$, we define the following Lagrange function:

$$
\mathrm{L}(x, \lambda, \mu)=f(x)+\frac{l}{\lambda^{t} l} \mu^{t} g(x), \quad \forall x \in S, \lambda \in K^{*}, \mu \in Q^{*} .
$$

Theorem 4.1. (Weak Duality) Let $x$ be feasible for (NVOP) and $(y, \lambda, \mu)$ be feasible for (NVUD). If $f$ is $K$-nonsmooth $(F, \rho)$-convex of order $k$ with respect to $\omega$ at $y$ on $S_{0}$ and $g$ is $Q$-nonsmooth $(F, \sigma)$-convex of order $k$ with respect to the same $\omega$ at $y$ on $S_{0}$, with $\lambda>0, \mu \geq 0$ and

$$
\lambda^{t} \rho+\mu^{t} \sigma \geq 0
$$

then,

$$
f(y)+\frac{l}{\lambda^{t} l}(1-\delta) \mu^{t} g(y)-f(x) \notin \operatorname{int} K .
$$

Proof: Assume on the contrary that

$$
f(y)+\frac{l}{\lambda^{t} l}(1-\delta) \mu^{t} g(y)-f(x) \in \operatorname{int} K .
$$

Since $(y, \lambda, \mu)$ is feasible for (NVUD), therefore by (2), there exist $\bar{A} \in \partial f(y)$ and $\bar{B} \in \partial g(y)$ such that

$$
\bar{A}^{t} \lambda+\bar{B}^{t} \mu=0 \text {. }
$$

Since $f$ is $K$-nonsmooth $(F, \rho)$-convex of order $k$ with respect to $\omega$ at $y$ on $S_{0}$, we have

$$
f(x)-f(y)-F(x, y ; \bar{A})-\rho\|\omega(x, y)\|^{k} \in K .
$$

Adding (21) and (23), we obtain

$$
\frac{l}{\lambda^{t} l}(1-\delta) \mu^{t} g(y)-F(x, y ; \bar{A})-\rho\|\omega(x, y)\|^{k} \in \operatorname{int} K .
$$


As $\lambda \in K^{*} \backslash\{0\}$, we get

$$
(1-\delta) \mu^{t} g(y)-\lambda^{t} F(x, y ; \bar{A})-\lambda^{t} \rho\|\omega(x, y)\|^{k}>0 .
$$

Also, since $g$ is $Q$-nonsmooth $(F, \sigma)$-convex of order $k$ with respect to $\omega$ at $y$ on $S_{0}$ and $\mu \in Q^{*}$, we have

$$
\mu^{t}\left[g(x)-g(y)-F(x, y ; \bar{B})-\sigma\|\omega(x, y)\|^{k}\right] \geq 0 .
$$

Adding (24) and (25), we get

$$
\mu^{t} g(x)-\delta \mu^{t} g(y)>\lambda^{t} F(x, y ; \bar{A})+\mu^{t} F(x, y ; \bar{B})+\left(\lambda^{t} \rho+\mu^{t} \sigma\right)\|\omega(x, y)\|^{k}
$$

or,

$$
\mu^{t} g(x)-\delta \mu^{t} g(y)>\sum_{i=1}^{m} \lambda_{i} F\left(x, y ; \bar{A}_{i}\right)+\sum_{j=1}^{p} \mu_{j} F\left(x, y ; \bar{B}_{j}\right)+\left(\lambda^{t} \rho+\mu^{\prime} \sigma\right)\|\omega(x, y)\|^{k} .
$$

Using sublinearity of $F$ under the assumption that $\lambda>0$ and $\mu \geq 0$, together with (22), (1) and (20), we obtain

$$
\delta \mu^{t} g(y)<\mu^{t} g(x) .
$$

As $x \in S_{0},-g(x) \in Q$ and $\mu \in Q^{*}$, so that $\mu^{t} g(x) \leq 0$ and we have $\delta \mu^{t} g(y)<0$.

This contradicts the feasibility of $(y, \lambda, \mu)$, hence the result.

Theorem 4.2. (Weak Duality) Let $x$ be feasible for (NVOP) and $(y, \lambda, \mu)$ be feasible for (NVUD) with $\lambda>0$ and $\mu \geq 0$. Suppose the following conditions hold:

(i) If $\delta=0, \rho \in K, \mathrm{~L}(., \lambda, \mu)$ is $K$-nonsmooth $(F, \rho)$-pseudoconvex type II of order $k$ with respect to $\omega$ at $y$ on $S_{0}$, and

(ii) If $\delta=1, \lambda^{t} \rho+\mu^{t} \sigma \geq 0, f$ is $K$-nonsmooth $(F, \rho)$-pseudoconvex type II of order $k$ with respect to $\omega$ at $y$ on $S_{0}$ and $g$ is $Q$-nonsmooth $(F, \sigma)$-quasiconvex type I of order $k$ with respect to $\omega$ at $y$ on $S_{0}$.

Then, we have

$$
f(y)+\frac{l}{\lambda^{t} l}(1-\delta) \mu^{t} g(y)-f(x) \notin \operatorname{int} K .
$$

Proof: Case (i): Let $\delta=0$ and on the contrary assume that,

$$
f(y)+\frac{l}{\lambda^{t} l} \mu^{t} g(y)-f(x) \in \operatorname{int} K .
$$

Since $x$ is feasible for (NVOP) and $\mu \in Q^{*}$, therefore $-\mu^{t} g(x) \geq 0$. Further, $l \in \operatorname{int} K$ so that

$$
-\frac{l}{\lambda^{t} l} \mu^{t} g(x) \in K \text {. }
$$

Adding (26) and (27), we get

$$
\left[\left\{f(y)+\frac{l}{\lambda^{t} l} \mu^{t} g(y)\right\}-\left\{f(x)+\frac{l}{\lambda^{t} l} \mu^{t} g(x)\right\}\right] \in \operatorname{int} K .
$$

That is,

$$
-[\mathrm{L}(x, \lambda, \mu)-\mathrm{L}(y, \lambda, \mu)] \in \operatorname{int} K .
$$

As $\mathrm{L}(., \lambda, \mu)$ is $K$-nonsmooth $(F, \rho)$-pseudoconvex type II of order $k$ with respect to $\omega$, we have for all $C=\left(C_{1}, \cdots, C_{m}\right)^{t} \in \partial \mathrm{L}(y, \lambda, \mu)$ 


$$
F(x, y ; C)+\rho\|\omega(x, y)\|^{k} \in-\operatorname{int} K .
$$

Since, $\lambda \in K^{*} \backslash\{0\}$, we get

$$
\lambda^{t} F(x, y ; C)+\lambda^{t} \rho\|\omega(x, y)\|^{k}<0,
$$

or

$$
\sum_{i=1}^{m} \lambda_{i} F\left(x, y ; C_{i}\right)+\lambda^{t} \rho\|\omega(x, y)\|^{k}<0,
$$

so that

$$
F\left(x, y ; \lambda^{t} C\right)+\lambda^{t} \rho\|\omega(x, y)\|^{k}<0 .
$$

Now, since $(y, \lambda, \mu)$ is feasible for (NVUD),

$$
\begin{aligned}
0 \in \partial\left(\lambda^{t} f+\mu^{t} g\right)(y) & =\partial\left(\lambda^{t} f+\frac{\lambda^{t} l}{\lambda^{t} l} \mu^{t} g\right)(y) \\
& =\partial\left(\sum_{i=1}^{m} \lambda_{i} f_{i}+\frac{1}{\lambda^{t} l} \sum_{i=1}^{m} \lambda_{i} l_{i} \mu^{t} g\right)(y) \\
& =\partial\left\{\sum_{i=1}^{m} \lambda_{i}\left(f_{i}+\frac{l_{i}}{\lambda^{t} l} \mu^{t} g\right)\right\}(y) \\
& \subseteq \sum_{i=1}^{m} \lambda_{i} \partial\left(f_{i}+\frac{l_{i}}{\lambda^{t} l} \mu^{t} g\right)(y) \\
& =\partial \mathrm{L}(y, \lambda, \mu)^{t} \lambda .
\end{aligned}
$$

Therefore, there exists $\hat{C} \in \partial \mathrm{L}(y, \lambda, \mu)$ such that $\lambda^{t} \hat{C}=0$. Substituting in (28) and then using (1), we get

$$
\lambda^{t} \rho\|\omega(x, y)\|^{k}<0,
$$

which is a contradiction, as $\lambda \in K^{*} \backslash\{0\}, \rho \in K$ and norm is a non-negative function.

Case (ii): Let $\delta=1$, then we have to prove that

$$
f(y)-f(x) \notin \operatorname{int} K .
$$

Let if possible,

$$
f(y)-f(x) \in \operatorname{int} K
$$

Since $f$ is $K$-nonsmooth $(F, \rho)$-pseudoconvex type II of order $k$ with respect to $\omega$ at $y$ on $S_{0}$, we have

$$
-\left\{F(x, y ; \bar{A})+\rho\|\omega(x, y)\|^{k}\right\} \in \operatorname{int} K .
$$

As $\lambda \in K^{*} \backslash\{0\}$, we get

$$
\lambda^{t} F(x, y ; \bar{A})+\lambda^{t} \rho\|\omega(x, y)\|^{k}<0 .
$$

Since $x$ is feasible for (NVOP) and $(y, \lambda, \mu)$ is feasible for (NVUD), we have

$$
\mu^{t}\{g(x)-g(y)\} \leq 0 .
$$

If $\mu \neq 0$, (30) implies $g(x)-g(y) \notin$ int $Q$. 
As $g$ is $Q$-nonsmooth $(F, \sigma)$-quasiconvex type I of order $k$ with respect to $\omega$ at $y$ on $S_{0}$, we get

$$
-\left\{F(x, y ; \bar{B})+\sigma\|\omega(x, y)\|^{k}\right\} \in Q .
$$

Since $\mu \in Q^{*}$, we have

$$
\mu^{t} F(x, y ; \bar{B})+\mu^{t} \sigma\|\omega(x, y)\|^{k} \leq 0
$$

If $\mu=0$, then also (31) holds.

Since $(y, \lambda, \mu)$ is feasible for (NVUD), by Remark 3.1, there exist $\bar{A} \in \partial f(y)$ and $\bar{B} \in \partial g(y)$ such that (22) holds.

Adding (29) and (31), we get

$$
\lambda^{t} F(x, y ; \bar{A})+\mu^{t} F(x, y ; \bar{B})+\left(\lambda^{t} \rho+\mu^{t} \sigma\right)\|\omega(x, y)\|^{k}<0,
$$

or

$$
\sum_{i=1}^{m} \lambda_{i} F\left(x, y ; \bar{A}_{i}\right)+\sum_{j=1}^{p} \mu_{j} F\left(x, y ; \bar{B}_{j}\right)+\left(\lambda^{t} \rho+\mu^{t} \sigma\right)\|\omega(x, y)\|^{k}<0 .
$$

Using sublinearity of $F$ with the fact that $\lambda>0$ and $\mu \geq 0$ and then using (22) and (1), we obtain

$$
\left(\lambda^{t} \rho+\mu^{t} \sigma\right)\|\omega(x, y)\|^{k}<0 .
$$

This contradicts the assumption that $\lambda^{t} \rho+\mu^{t} \sigma \geq 0$, hence the result.

Theorem 4.3. (Strong Duality) Let $\bar{x}$ be a weak minimizer of order $k$ with respect to $\omega$ for (NVOP) with $S=\mathbf{R}^{n}$, at which Slater-type cone constraint qualification holds. Then there exist $\bar{\lambda} \in K^{*} \backslash\{0\}, \bar{\mu} \in Q^{*}$ such that $(\bar{x}, \bar{\lambda}, \bar{\mu})$ is feasible for (NVUD). Further, if the conditions of Weak Duality Theorem 4.1 (Theorem 4.2) hold for all feasible $x$ for (NVOP) and all feasible $(y, \lambda, \mu)$ for (NVUD), then $\bar{x}$ is a weak maximizer of order $k$ with respect to $\omega$ for (NVUD).

Proof: As $\bar{x}$ is a weak minimizer of order $k$ with respect to $\omega$ for (NVOP), by Theorem 3.2 there exist $\bar{\lambda} \in K^{*} \backslash\{0\}, \bar{\mu} \in Q^{*}$ such that

$$
\begin{gathered}
0 \in \partial\left(\bar{\lambda}^{t} f+\bar{\mu}^{t} g\right)(\bar{x}), \\
\bar{\mu}^{t} g(\bar{x})=0 .
\end{gathered}
$$

Since $\delta \in\{0,1\}$, Equations (32) and (33) can be written as

$$
\begin{gathered}
0 \in \partial\left(\bar{\lambda}^{t} f+\bar{\mu}^{t} g\right)(\bar{x}), \\
\delta \bar{\mu}^{t} g(\bar{x})=0 .
\end{gathered}
$$

Thus, $(\bar{x}, \bar{\lambda}, \bar{\mu})$ is a feasible solution for (NVUD). Further, if $(\bar{x}, \bar{\lambda}, \bar{\mu})$ is not a weak maximizer of order $k$ with respect to $\omega$ for (NVUD), then for any $\beta \in \operatorname{int} K$, there exists a feasible solution $(y, \lambda, \mu)$ of (NVUD) such that

$$
\left\{f(y)+\frac{l}{\lambda^{t} l}(1-\delta) \mu^{t} g(y)\right\}-\left\{f(\bar{x})+\frac{l}{\bar{\lambda}^{t} l}(1-\delta) \bar{\mu}^{t} g(\bar{x})\right\}-\beta\|\omega(y, \bar{x})\|^{k} \in \operatorname{int} K,
$$

or,

$$
f(y)+\frac{l}{\lambda^{t} l}(1-\delta) \mu^{t} g(y)-f(\bar{x})-\beta\|\omega(y, \bar{x})\|^{k} \in \operatorname{int} K .
$$


Since, $\beta \in \operatorname{int} K, \beta\|\omega(y, \bar{x})\|^{k} \in \operatorname{int} K$, so that we have

$$
f(y)+\frac{l}{\lambda^{t} l}(1-\delta) \mu^{t} g(y)-f(\bar{x}) \in \operatorname{int} K,
$$

which contradicts Theorem 4.1 (Theorem 4.2). Hence $(\bar{x}, \bar{\lambda}, \bar{\mu})$ is a weak maximizer of order $k$ with respect to $\omega$ for (NVUD).

\section{Conclusion}

In this paper, we introduced the concept of a higher-order (weak) minimizer for a nonsmooth vector optimization problem over cones. Furthermore, to study the new solution concept, we defined new generalized classes of cone-nonsmooth $(F, \rho)$-convex functions and established several sufficient optimality and duality results using these classes. The results obtained in this paper will be helpful in studying the stability and convergence analysis of iterative procedures for various optimization problems.

\section{References}

[1] Bector, C.R., Chandra, S. and Bector, M.K. (1988) Sufficient Optimality Conditions and Duality for a Quasiconvex Programming Problem. Journal of Optimization Theory and Applications, 59, 209-221.

[2] Mangasarian, O.L. (1969) Nonlinear Programming. McGraw-Hill, New York.

[3] Vial, J.P. (1983) Strong and Weak Convexity of Sets and Functions. Mathematics of Operations Research, 8, $231-259$. http://dx.doi.org/10.1287/moor.8.2.231

[4] Hanson, M.A. and Mond, B. (1982) Further Generalization of Convexity in Mathematical Programming. Journal of Information and Optimization Sciences, 3, 25-32. http://dx.doi.org/10.1080/02522667.1982.10698716

[5] Preda, V. (1992) On Efficiency and Duality for Multiobjective Programs. Journal of Mathematical Analysis and Applications, 166, 365-377. http://dx.doi.org/10.1016/0022-247X(92)90303-U

[6] Antczak, T. and Kisiel, K. (2006) Strict Minimizers of Order m in Nonsmooth Optimization Problems. Commentationes Mathematicae Universitatis Carolinae, 47, 213-232.

[7] Antczak, T. (2011) Characterization of Vector Strict Global Minimizers of Order 2 in Differentiable Vector Optimization Problems under a New Approximation Method. Journal of Computational and Applied Mathematics, 235, 49915000. http://dx.doi.org/10.1016/j.cam.2011.04.029

[8] Cromme, L. (1978) Strong Uniqueness: A Far Criterion for the Convergence Analysis of Iterative Procedures. Numerische Mathematik, 29, 179-193. http://dx.doi.org/10.1007/BF01390337

[9] Studniarski, M. (1989) Sufficient Conditions for the Stability of Local Minimum Points in Nonsmooth Optimization. Optimization, 20, 27-35. http://dx.doi.org/10.1080/02331938908843409

[10] Bhatia, G. and Sahay, R.R. (2013) Strict Global Minimizers and Higher-Order Generalized Strong Invexity in Multiobjective Optimization. Journal of Inequalities and Applications, 2013, 31. http://dx.doi.org/10.1186/1029-242X-2013-31

[11] Clarke, F.H. (1983) Optimization and Nonsmooth Analysis. Wiley, New York.

[12] Studniarski, M. (1997) Characterizations of Strict Local Minima for Some Nonlinear Programming Problems. Nonlinear Analysis, Theory, Methods \& Applications, 30, 5363-5367. http://dx.doi.org/10.1016/S0362-546X(97)00352-0

[13] Ward, D.W. (1994) Characterizations of Strict Local Minima and Necessary Conditions for Weak Sharp Minima. Journal of Optimization Theory and Applications, 80, 551-571. http://dx.doi.org/10.1007/BF02207780

[14] Nahak, C. and Mohapatra, R.N. (2012) Nonsmooth $\rho-(\eta, \theta)$-Invexity in Multiobjective Programming Problems. Optimization Letters, 6, 253-260. http://dx.doi.org/10.1007/s11590-010-0239-1

[15] Craven, B.D. (1989) Nonsmooth Multiobjective Programming. Numerical Functional Analysis and Optimization, 10, 49-64. http://dx.doi.org/10.1080/01630568908816290

[16] Cambini, R. and Carosi, L. (2010) Mixed Type Duality for Multiobjective Optimization Problems with Set Constraints. In: Jimènez, M.A., Garzón, G.R. and Lizana, A.R., Eds., Optimality Conditions in Vector Optimization, Bentham Science Publishers, Sharjah, 119-142. 
Scientific Research Publishing (SCIRP) is one of the largest Open Access journal publishers. It is currently publishing more than 200 open access, online, peer-reviewed journals covering a wide range of academic disciplines. SCIRP serves the worldwide academic communities and contributes to the progress and application of science with its publication.

Other selected journals from SCIRP are listed as below. Submit your manuscript to us via either submit@scirp.org or Online Submission Portal.
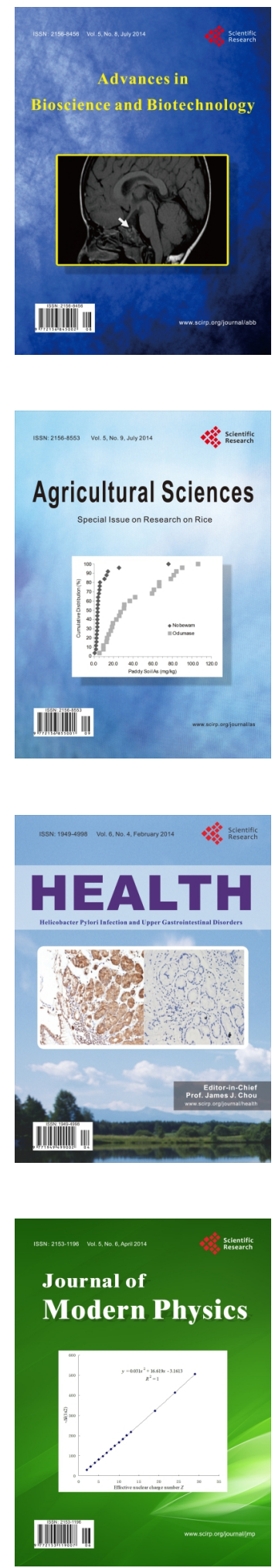
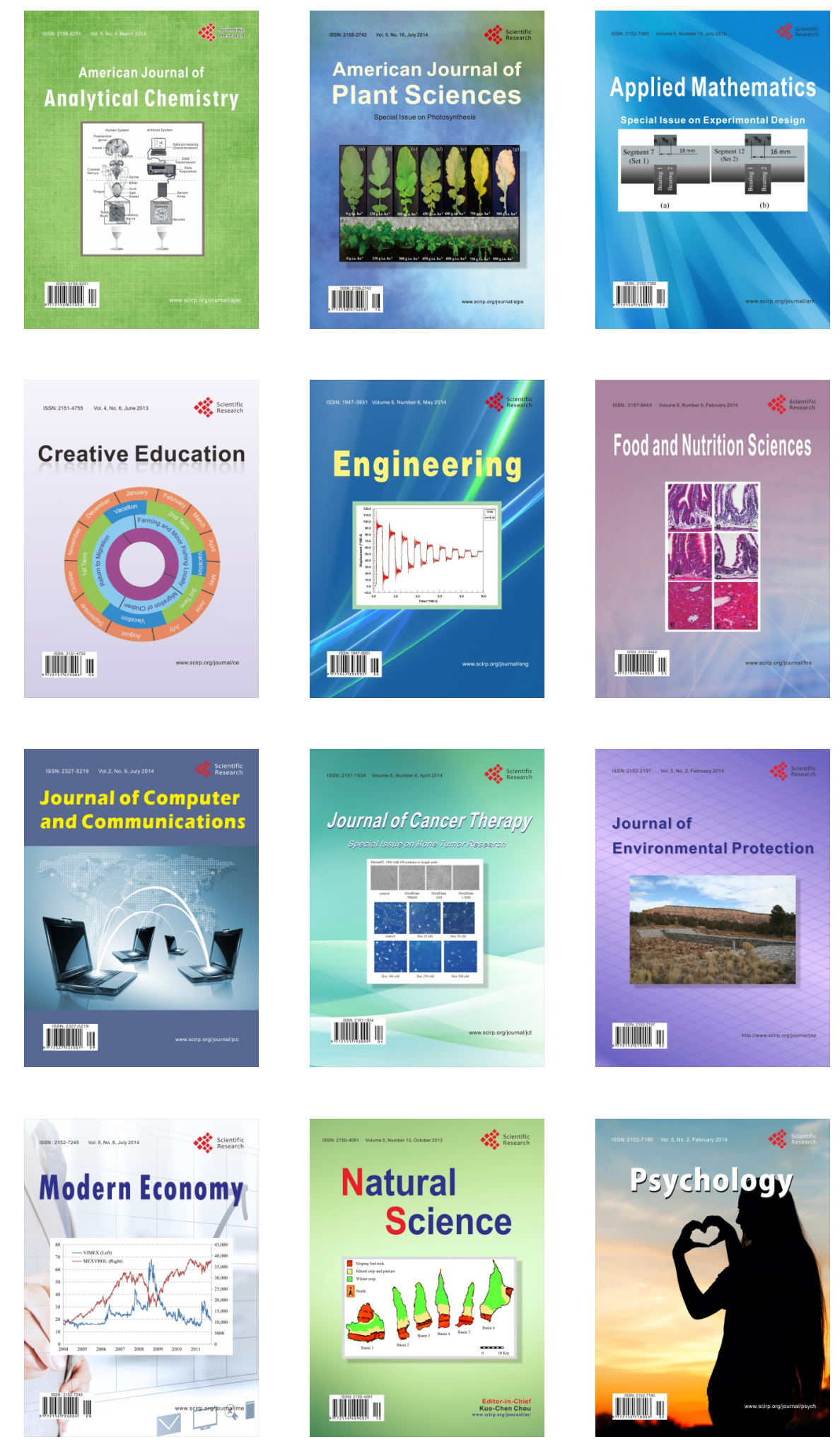